\title{
A Study of Electrospray Ionization Emitters with Differing Geometries with Respect to Flow Rate and Electrospray Voltage
}

\author{
Brent R. Reschke, ${ }^{2}$ Aaron T. Timperman ${ }^{1}$ \\ ${ }^{1}$ Engineer Research \& Development Center (ERDC)-Construction Engineering Research Laboratory (CERL), 2902 Newmark \\ Dr., Champaign, IL 61826, USA \\ ${ }^{2}$ C. Eugene Bennet Department of Chemistry, West Virginia University, Morgantown, WV 26506, USA
}

\begin{abstract}
The performance of several electrospray ionization emitters with different orifice inside diameters (i.d.s), geometries, and materials are compared. The sample solution is delivered by pressure driven flow, and the electrospray ionization voltage and flow rate are varied systematically for each emitter investigated, while the signal intensity of a standard is measured. The emitters investigated include a series of emitters with a tapered outside diameters (o.d.) and unaltered i.d.s, a series of emitters with tapered o.d.s and i.d.s, an emitter with a monolithic frit and a tapered o.d., and an emitter fabricated from polypropylene. The results show that for the externally etched emitters, signal was nearly independent of i.d. and better ion utilization was achieved at lower flow rates. Furthermore, emitters with a $50 \mu \mathrm{m}$ i.d. and an etched o.d. produced about 1.5 times more signal than etched emitters with smaller i.d.s and about 3.5 times more signal than emitters with tapered inner and outer dimensions. Additionally, the work presented here has important implications for applications in which maximizing signal intensity and reducing frictional resistance to flow are necessary. Overall, the work provides an initial assessment of the critical parameters that contribute to maximizing the signal for electrospray ionization sources interfaced with pressure driven flows.
\end{abstract}

Key words: Electrospray ionization, Etched open-tubular emitter, Polymeric emitter, Pulled tip emitter, Electrospray voltage, Flow rate, Reserpine

\section{Introduction}

E lectrospray ionization (ESI) is a commonly used ionization method for mass spectrometry (MS). ESI is the method of choice for producing gas phase ions from solution because it is a very soft ionization method and is useful for the ionization of larger molecules or biomolecules. ESI-MS

Electronic supplementary material The online version of this article (doi:10.1007/s13361-011-0251-4) contains supplementary material, which is available to authorized users.

Correspondence to: Aaron T. Timperman; e-mail: aaron.timperman@usace. army.mil can be used for the analysis of complex mixtures [1-5] and is commonly used to couple separation techniques, such as high performance liquid chromatography (HPLC) [6-9], capillary electrophoresis (CE) [10-21], or microchannel electrophoresis (ME) [22-29] with MS. Many sample introduction methods, particularly those that employ separations, rely on pressure driven flow for sample introduction. For optimal sample utilization, determination of the parameters that provide the highest signal intensity is crucial. Many previous studies have been performed to optimize different parameters that affect the ESI signal, like sheath flow [10, 12, 20], nebulizing gas [13, 16], buffer systems $[20,30]$, and ESI voltage [31, 32]. Likewise, ESI emitters have been extensively studied because the characteristics of 
the emitter, such as the emitter i.d., the surface area of the emitter orifice, and the hydrophobicity, have a great impact on the observed ESI signal; in addition to influencing the working parameters of the system, such as flow rate or ESI voltage [33].

The emitter i.d. has a direct impact on many of the other factors that affect the electrospray process and therefore, require careful consideration. As solution emanates from an emitter and is electrosprayed, the solution will have a natural tendency to evenly spread along the surface from which it stems. The amount the solution spreads is, in part, determined by the hydrophobic character of the emitter surface; a more hydrophobic emitter will exhibit less wet ability [34] and create a more distinct droplet at the tip. As the volume of solution at the tip increases it becomes harder to electrospray at a given voltage. Therefore, hydrophobic emitters with smaller i.d.s will, generally, have a smaller surface area and accordingly will constrain the solution to a smaller volume as it flows from the emitter at a given flow rate, either in the Taylor cone or as a droplet on the tip [35]. Because more hydrophobic emitters minimize the volume of solution accumulating at the emitter tip, the voltage required to bring about the onset of the electrospraying process is also reduced [33].

Small i.d. emitters have also been shown to create droplets with smaller dimensions compared with the droplets produced by emitters with larger i.d.s [36-39]. Wilm and Mann predicted $180 \mathrm{~nm}$ diameter droplets, that statistically contain about 1 molecule per droplet when used with an analyte concentration of $0.5 \mathrm{pmol} / \mu \mathrm{L}$ [36]. Furthermore, the droplets produced by smaller i.d. emitters also have a higher charge density, which ultimately leads to increased ionization efficiency and produce better signal [40-42]. Li and Cole noted that spectra produced from narrow i.d. emitters also showed higher average charge states, presumably because of the increased charge density on the droplets [41].

Emitters with smaller i.d.s also have been shown to have increased signal intensity compared to emitters with larger $i$. d.s $[7,12,21,37,38,40,41,43,44]$. The increased signal intensity observed with smaller i.d. emitters results from a decrease in the amount of background electrolyte exiting the orifice and thereby allows a greater portion of the analyte to be converted to the gas phase. Increased gas phase analyte ion production is caused by decreased competition for charge between the analyte and solvent thus increasing ionization efficiency [21, 45].

Smaller i.d. emitters also allow lower flow rates to be used in addition to producing smaller droplets and minimizing the volume of solution accumulating at the tip. Lower flow rates also help increase the sensitivity in ESI because the background electrolyte is reduced resulting in higher ionization efficiencies [21, 36, 43, 46-49]. Furthermore, when lower flow rates are used for ESI in applications such as LC-MS or CE-MS sample bands are constrained to a smaller volume thus minimizing sample dilution and as a result show improved limits of detection (LODs) [36, 37, 50]. A general trend reported in many references is that at moderate to high flow rates, electrospray is concentration sensitive and the flow rate has little affect on the signal intensity [7, 21, 32, 43, 46, 47, 51-53]. At very low flow rates, on the order of $10 \mathrm{~nL} / \mathrm{min}$, some signal intensity to flow rate dependence is observed, but the increase in signal is less than proportional to the increase in the mass of the sample consumed [7, 21, 49]. This feature has important implications for mass-limited samples, such as those typically encountered in proteomics. When the analyte is constrained to a smaller volume as it is electrosprayed, using lower flow rates and narrow i.d. emitters, the signal will be maximized and the probability of detecting low abundance analytes is increased.

To improve detection and optimize sample utilization, previous reports have compared different ESI emitters to determine, which provides the best signal intensity. Commonly investigated ESI emitters are emitters that have tapered inner and outer dimensions. These emitters are created by heating a fused silica capillary and pulling to form a tapered tip. Emitters created using this method will be referred to as "pulled-tapered emitters" (PTE). A number of important early studies of ESI emitters employed nanospray, which does not use pressure driven flow, but relies on removal of the solution by the electrospray process to drive the sample through the emitter. Using the nanospray approach, Valaskovic et al. compared the signal observed from gold-coated PTEs with different orifice i. d.s fabricated from fused silica capillary [54]. Their results showed improved LODs when using $5 \mu \mathrm{m}$ i.d. capillaries compared with $20 \mu \mathrm{m}$ i.d. capillaries. Kelly et al. also compared different gold-coated PTE emitters that were fabricated by attaching a weight to one end of a fused silica capillary and heating the other end to form a tapered emitter [18]. In this work, the analyte response for three gold-coated emitters with different tip i.d.s was compared. Their results showed that a $13 \mu \mathrm{m}$ tip i.d. produced an analyte response three times higher than the response from a $30 \mu \mathrm{m}$ tip i.d.. Finally, Ishihama et al. compared the signal observed from a series of stainless steel emitters, ranging from and $20 \mu \mathrm{m}$ i.d. $/ 70 \mu \mathrm{m}$ o.d. to $76 \mu \mathrm{m}$ i.d./ $305 \mu \mathrm{m}$ o.d., with a geometry similar to a PTE as a function of flow rate, voltage, and i.d. [43]. Similar to results reported by others, their results showed that smaller i.d. emitters produced more stable spray and higher $\mathrm{S} / \mathrm{N}$ ratios at lower flow rates.

While smaller i.d. emitters have many clear advantages, there are several drawbacks that must also be considered. A major difficulty associated with smaller i.d. emitters when used with $\mathrm{CE}$ or $\mathrm{ME}$ is their resistance to flow. As the i.d. of the emitter decreases, the resistance to volumetric flow increases as a function of $r^{4}$, where $r$ is the inner radius of the emitter [55]. This resistance to flow results in increased backpressure, which is of little concern for HPLC interfaces, but can substantially reduce the flow rate and can cause significant band broadening in systems that rely on electroosmotic flow (EOF). Another issue with smaller i.d. emitters is their susceptibility to clogging by small particulates. Once an emitter becomes clogged, ESI ceases and the emitter must be replaced before analysis can continue. Tip replacement usually results in sample loss and requires time for replacement. Furthermore, tips that have a propensity to clog are not suited for use with automated systems. 
To reduce the problems associated with emitter fouling, Kelly et al. etched the o.d. of fused silica capillaries with hydrofluoric acid (HF) to create a tapered o.d. while passing water through the capillary to prevent etching of the i.d. [44]. Emitters of this type will be referred to as etched opentubular emitters (EOEs). Because the i.d. of these emitters is unchanged they are less prone to clogging, while still minimizing the surface area of the o.d.. In this work, a $5 \mu \mathrm{m}$ EOE was shown to generate reproducible signal over a range of ESI voltages at both 5 and $50 \mathrm{~nL} / \mathrm{min}$. The authors also provided a qualitative comparison of emitter fouling between EOEs and PTES and found that the EOEs had significantly less fouling compared with the PTEs. Furthermore, they noted that when EOE emitter fouling did occur, it was usually not from clogging but rather analyte precipitation or damage from mishandling.

This paper provides a relative comparison of the signal intensity produced by a few common types of electrospray emitters for pressure driven flow over a range of flow rates and electrospray voltages. A range of emitter geometries and materials is explored by using PTEs, EOEs, a monolithic emitter, and a molded polypropylene tip. PTEs with orifice $\mathrm{i}$. d.s of 5, 8, and $15 \mu \mathrm{m}$ and EOEs with i.d.s of 10, 20, 30, and $50 \mu \mathrm{m}$ were investigated. The electrospray voltage was varied systematically from 0.75 to $4.0 \mathrm{kV}$ while the flow rate was varied from 10 to $500 \mathrm{~nL} / \mathrm{min}$. The results show the combinations of flow rates and ESI potentials, which provide the greatest signal intensity and ion utilization efficiency for each emitter. Overall, the best performance is observed for EOEs, which consistently produced the highest signal intensities and a large stable ESI range. Surprisingly, the greatest signal intensity is observed for the largest orifice i.d.s investigated in this study. It is anticipated that these results will be useful for improving future emitter designs.

\section{Experimental}

\section{Chemicals and Reagents}

Unless otherwise noted, all chemicals were purchased from Sigma-Aldrich (Milwaukee, WI, USA). The analyte solution was $50 \%$ methanol $50 \%$ deionized water with $0.1 \mathrm{M}$ acetic acid and $0.1 \mathrm{mg} / \mathrm{mL}$ reserpine (MW: 608.68). The water was dispensed from a Barnstead International NanoPure Infinity (Dubuque, IA, USA) with a nominal resistivity of 18.3 $\mathrm{M} \Omega^{*} \mathrm{~cm}$. Concentrated $(47 \%-51 \%)$ hydrofluoric acid (HF) was purchased from Fisher Scientific (Fair Lawn, NJ, USA) and was used to etch the fused silica capillaries.

\section{Electrospray Emitters}

Pictures of the different types of emitters used for this work are shown in Figure 1. The EOEs were created using a procedure similar to one developed by Kelly et al. [44]. Briefly, the capillary was cleaved and polished to a flat edge, and then about $1 \mathrm{~cm}$ of the polyimide coating was removed
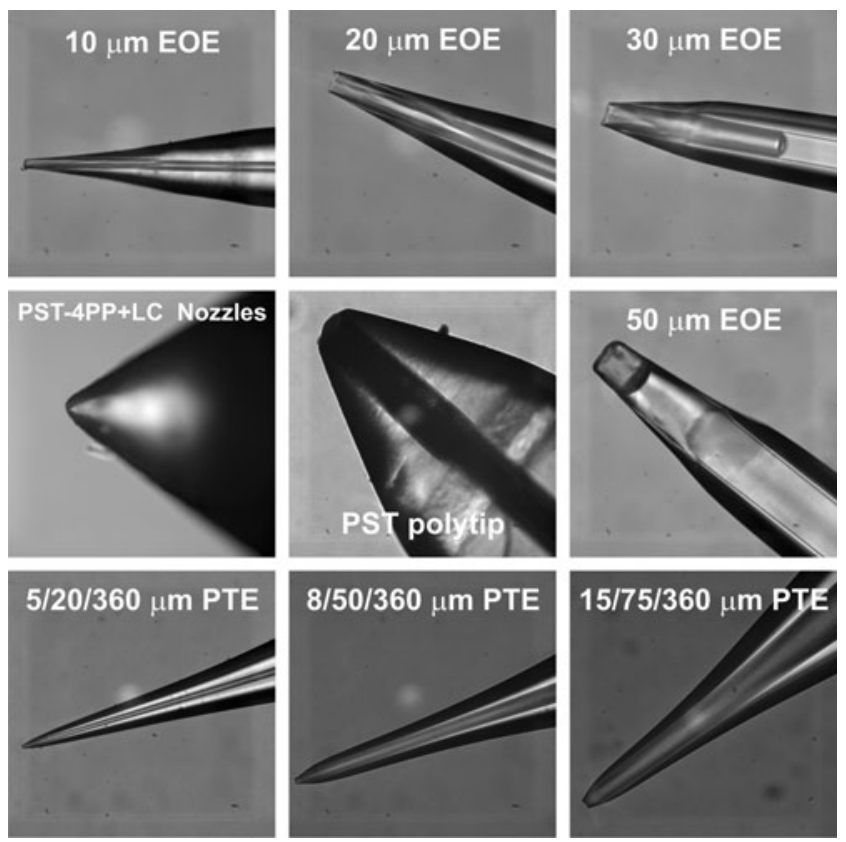

Figure 1. Photomicrographs of the different ESI emitters used in this study are shown. The top three images are different i.d. EOEs. The second row shows the molded polymeric nozzle (PST-4PP + LC nozzles), the monolithic emitter (PST polytip), and the $50 \mu \mathrm{m}$ EOE. Finally, the last three are the different PTEs. All images were acquired using a $40 \times$ objective

from the polished end. Next, $5 \mathrm{~mm}$ of the exposed capillary was submerged in the concentrated HF and the capillary was etched until the silica was no longer in contact with the HF while passing deionized water through the capillary at a flow rate of $0.1 \mu \mathrm{L} / \mathrm{min}$ using a KD Scientific syringe pump (model 7803118; Holliston, MA, USA). Please note, HF is very hazardous and extreme care should be exercised when $H F$ is used. Accepted methods for handling and use of HF should be followed because exposure to vapor and skin contact is very dangerous. In addition, calcium gluconate should be kept nearby for treatment in case of an emergency exposure. The capillary used was purchased from Polymicro Technologies (Phoenix, AZ, USA) with i.d.s of 10, 20, 30, 50,100 , and $200 \mu \mathrm{m}$ and all had an o.d. of $360 \mu \mathrm{m}$.

Three sets of PTEs were purchased from New Objective (Woburn, MA, USA). The first set of PicoTip Emitters, SilicaTip FS360-75-15 (15/75/360 $\mu \mathrm{m}$ PTEs), had a tip i.d. of $15 \mu \mathrm{m}$, an i.d. of $75 \mu \mathrm{m}$, and an o.d. of $360 \mu \mathrm{m}$. The second set was PicoTip Emitters, SilicaTip FS360-50-8 (8/50/360 $\mu \mathrm{m}$ PTEs), had a tip i.d. of $8 \mu \mathrm{m}$, an i.d. of $50 \mu \mathrm{m}$, and an o.d. of $360 \mu \mathrm{m}$. The third set contained PicoTip Emitters, SilicaTip FS360-20-5 (5/20/360 $\mu \mathrm{m}$ PTEs), and had a $5 \mu \mathrm{m}$ tip i.d., a $20 \mu \mathrm{m}$ i.d., and a $360 \mu \mathrm{m}$ o.d.

Both polymeric and monolithic emitters were purchased from Phoenix S \& T (Chester, PA, USA). PST-polytip emitters are made with a silica monolith [56] and an externally tapered tip having an i.d. of $50 \mu \mathrm{m}$ and an o.d. of $360 \mu \mathrm{m}$. A set of PST-4PP+LC nozzles were also 
purchased from Phoenix $\mathrm{S} \& \mathrm{~T}$, and these molded polypropylene nozzles have a nominal tip i.d. of $20 \mu \mathrm{m}$. The molded polypropylene nozzles were connected to a transfer capillary, which introduces a dead volume of $\sim 30 \mathrm{~nL}$ at the capillary/nozzle interface. All commercially available emitters were used as received.

\section{Instrumental Setup}

A schematic of the experimental setup is diagrammed in Supplemental Material 1. A ThermoFinnigan LCQ DECA XP (Waltham, MA, USA) ion trap mass spectrometer was used to obtain the mass spectra, and an Agilent 1100 series binary HPLC pump (Santa Clara, CA, USA) was used to pump the analyte solution through a PEEK microfilter into a PEEK microcross both from Upchurch Scientific (Oak Harbor, WA, USA). The other arms of the cross contained the MS electrode, the ESI emitter, and a waste capillary. The waste capillary was used to split the flow of the incoming solution between the ESI emitter and the waste capillary. A split ratio of 100:1, defined as the amount coming into the cross from the HPLC pump to the amount exiting the ESI emitter, was used to improve accuracy at low flow rates, and allow flow rates as low as $10 \mathrm{~nL} / \mathrm{min}$ to be investigated. Consequently, as the different spray emitters were investigated, the waste capillary was changed to maintain the split ratio. Each emitter that was investigated was cleaved to a length of $3 \mathrm{~cm}$ and tightened in place in the PEEK cross. Next, the emitter was centered in front of the MS ion transfer capillary $1 \mathrm{~mm}$ from the inlet using a Newport (Irvine, CA, USA) MT-XYZ compact stage. The distance was measured using a ruler built in-house as depicted in Figure S1 (Supplemental material).

To collect the data, both the ESI voltage and sample introduction flow rate were controlled automatically. The MS was controlled with Xcalibur ver. 1.3 SR1 (Waltham, MA, USA). Both full scans over the range of 150 to $1000 \mathrm{~m} / z$ and zoom scans over the range of 608 to $610 \mathrm{~m} / z$ were collected by toggling between the full scan and the zoom scan for $5 \mathrm{~min}$ at each voltage step. The ESI voltage was started at $0.75 \mathrm{kV}$ and was incremented in steps of $0.25 \mathrm{kV}$ up to a final voltage of $4 \mathrm{kV}$ for a total of $70 \mathrm{~min}$, while the flow rate was held constant. At the end of each series of potentials, the HPLC pump was ramped to the next flow rate and held constant for the next 70 min while the ESI potentials were scanned again. The flow rates examined were $10,30,50,80,100,150,200,250,300$, 400 , and $500 \mathrm{~nL} / \mathrm{min}$. After all the flow rates were studied, the data for the investigated emitter was exported from Xcalibur and averaged using Microsoft Excel. A total of five different emitters of each geometry were tested and the signal intensity values reported are the average signal values from each of the five runs. The flow rates were tested in both increasing and decreasing directions for every other emitter, i.e., for the first emitter the flow rates increased 10 to $500 \mu \mathrm{L} / \mathrm{min}$ and for the second emitter the flow rates decreased from 500 to $10 \mu \mathrm{L} / \mathrm{min}$. No difference was observed between the increasing and decreasing flow rate scans. The reported values were extracted from the zoom scans and the 3D Mesh plots were created using SigmaPlot 11.0 (Systat Software, Chicago, IL, USA) and CorelDraw 12.0 (Mountain View, CA, USA).

\section{Results and Discussion}

In this study, a sample solution of constant composition is delivered hydrodynamically to an ESI emitter and the signal intensity of reserpine is measured over a wide range flow rates and ESI voltages for different types of emitters. Overall, the results show little variation in the observed maximum signal intensity as a function of the emitter size, while a substantial variation is observed as a function of emitter geometry and material as shown in Figure 2. The signal intensities shown in Figure 2 are from the circled data points in Figures 3, 4, and 5, and are labeled with their corresponding flow rates. This graph shows little significant difference among the EOEs, as all of the values are nearly within one standard deviation of each other. Nevertheless, the $50 \mu \mathrm{m}$ EOE did provide the highest intensity, followed by the $10 \mu \mathrm{m}$ EOE with slightly lower values measured for the 20 and $30 \mu \mathrm{m}$ EOEs. Similarly, the PTE with the largest tip orifice $(15 / 75 / 360 \mu \mathrm{m})$ produced higher intensities compared with the two other PTEs investigated. Again, this result is in contrast to previous studies that have reported that the signal intensity increases with the use of smaller i.d. emitters, which has been attributed to production of smaller droplets by the smaller emitters [36-39] and greater ionization efficiencies [40-42]. The tabulated results in Figure 2 show increased signal intensity for the larger i.d.s; however, there is no clear trend with the EOEs because the signal intensity obtained with the 20 and $30 \mu \mathrm{m}$ emitters is less than the signal intensity obtained from the $10 \mu \mathrm{m}$ emitter. The cause of the lower maximum observed intensities with 20 and $30 \mu \mathrm{m}$ EOEs is currently unknown. It is possible that small experimental differences, such as the angle of the emitter orifice, could create such differences,

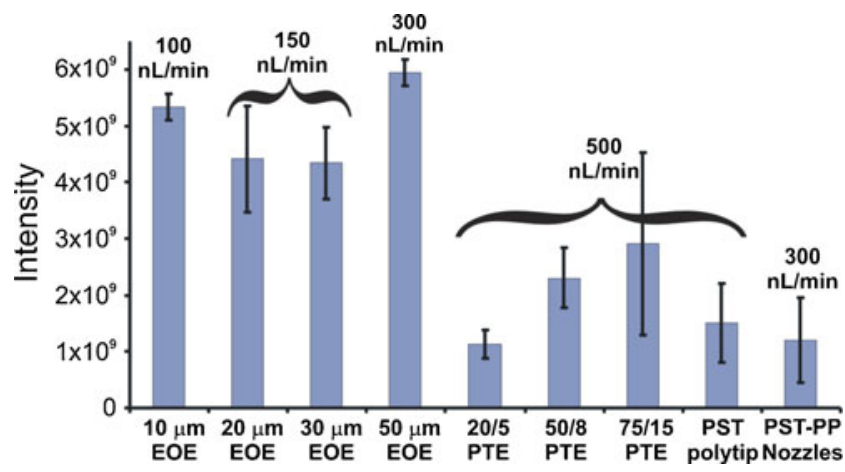

Figure 2. The highest observed average intensities for each emitter investigated are graphed and labeled with the corresponding flow rate values. The reported intensity values correspond to the circled data points in each of the 3D plots in Figures 3, 4, and 5 

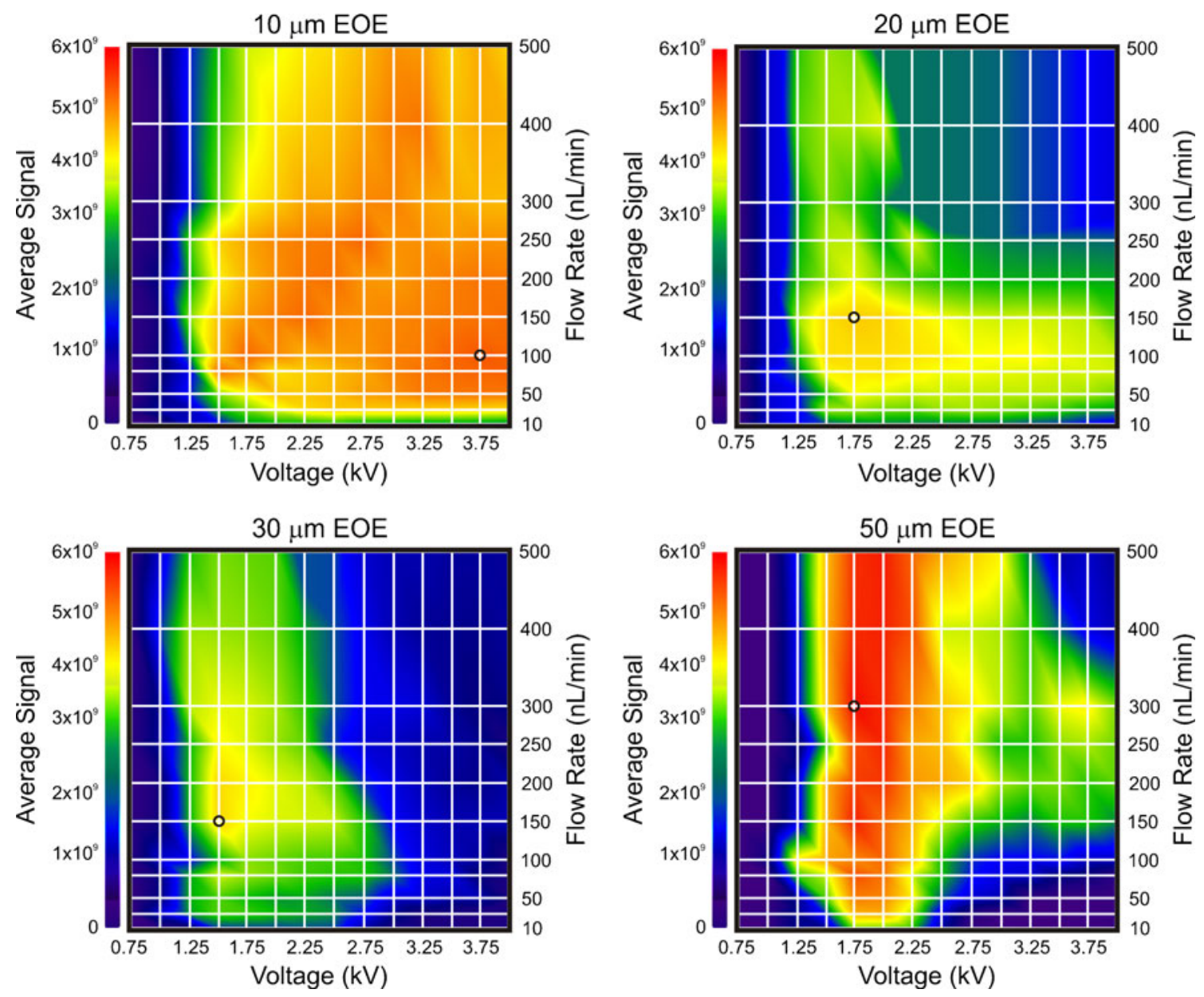

Figure 3. The 3D average intensity plots generated with the four different EOEs, having i.d.s of $10,20,30$, and $50 \mu \mathrm{m}$ are shown. The intensities for each plot are normalized to the highest intensities observed in the $50 \mu \mathrm{m}$ EOE 3D plot. The circled data points indicate the highest overall intensity for each emitter

which are fairly small when the standard deviations are considered. To reduce the effects of minor differences in emitter geometry and alignment, five replicates were produced for each emitter series and the plotted intensities are the averages of these five replicates. For each replicate, the ESI tip was replaced and centered with respect to the heated capillary bore with a distance of $1 \mathrm{~mm}$ separating the emitter from the end of the heated capillary.

To facilitate a direct comparison of the intensities for the plots within each figure, the intensity scale is held constant in Figures 3, 4, and 5, and the point of maximum intensity is circled on each plot. It should be noted that the color scale is quite sensitive to small intensity changes within each figure. Figures 3, 4, and 5 show the average intensity from the investigated emitters for a virtually unlimited sample volume. The ion utilization efficiency, obtained by dividing the average signal by the mass of sample consumed, confirms that lower flow rates produce greater signal for mass limited samples as shown in Figure 6.

The average signal intensity is shown for the EOE emitters as a function of flow rate and ESI voltage in Figure 3. At the lower ESI voltages $(0.75$ and $1 \mathrm{kV})$ for the
$10 \mu \mathrm{m}$ EOE investigated, the ESI signal is very low as the onset of ESI has not been reached. The onset of ESI occurs around $1.25 \mathrm{kV}$ and the emitter continues to spray at all other voltages investigated. At the lower flow rates of 10 and $30 \mathrm{~nL} / \mathrm{min}$, the signal intensity is fairly constant, while the highest signal is generally observed at the intermediate flow rates between 50 and $300 \mathrm{~nL} / \mathrm{min}$. Based on the data in the graph, near equivalent signal intensities are observed over a broad range of ESI voltages and flow rates. The average signal intensities observed in the $20 \mu \mathrm{m}$ EOE plot are, overall, lower when compared with the $10 \mu \mathrm{m}$ EOE plot. However, a similar trend is observed in that at the lower ESI voltages the signal is low and at about an ESI voltage of $1.25 \mathrm{kV}$ the onset of ESI occurs and continues for the other ESI voltages investigated. Likewise, flow rates from about 50 to $250 \mathrm{~nL} / \mathrm{min}$ generally showed the highest average intensities. The $30 \mu \mathrm{m}$ and $50 \mu \mathrm{m}$ EOE plots show a slightly different behavior. In these plots, the greatest signal intensity is observed shortly after the ESI onset, and after reaching a maximum the signal intensity decreases as the ESI voltage is increased. For the $30 \mu \mathrm{m}$ EOE, the maximum signal intensity is observed over the ESI voltage range of 1.25 to 
Figure 4. The average intensity 3D plots acquired with the three different PTEs, having orifice i.d.s of 5, 8, and $15 \mu \mathrm{m}$ are shown. The intensities for each plot are normalized to the highest intensities observed in the 15/75/360 $\mu \mathrm{m}$ PTE 3D plot. The circled data points indicate the highest overall intensity for each emitter, which are all observed at a flow rate of $500 \mathrm{~nL} / \mathrm{min}$

$2.25 \mathrm{kV}$ and a flow rate range from about 100 to $200 \mathrm{~nL} / \mathrm{min}$. The $50 \mu \mathrm{m}$ EOE plot again shows a trend similar to the $30 \mu \mathrm{m}$ EOE plot, with a slight shift in the maximum signal intensity to a higher ESI voltage with the greatest signal intensities observed over a range from about 1.50 to $2.50 \mathrm{kV}$. Overall, the $50 \mu \mathrm{m}$ EOEs produced the highest average intensities. The decrease in signal intensity as the voltage is increased with the $50 \mu \mathrm{m}$ EOE is likely caused by a transition from the cone-jet to the multi-jet mode, as previously reported [42, 57]. For the 30 and $50 \mu \mathrm{m}$ EOEs, it should be noted that the signal stability decreases and the standard deviation of the signal intensity increases as the ESI voltage increases. Likewise, the standard deviation of the signal intensity increased as the ESI voltage increased with the $20 \mu \mathrm{m}$ EOE, however to a significantly lesser extent. The signal stability in the $10 \mu \mathrm{m}$ EOE spectra contained little variability over the range of ESI voltages and flow rates and, consequently, appears to be the most robust. The decrease in the standard deviation of the signal intensity at the higher ESI voltages probably results from the production of smaller droplets with the smaller i.d. emitters.

The performance of the PTEs was characterized in a similar manner as shown in Figure 4. Clearly, the overall intensities from the PTEs are significantly lower than observed with the EOEs which provide signal intensities that are 2-fold greater or more. The highest intensities are recorded with the $15 / 75 / 360 \mu \mathrm{m}$ PTEs. However, these intensities are still about three times lower than the intensities observed with the $50 \mu \mathrm{m}$ EOEs at $200 \mathrm{~nL} / \mathrm{min}$. Slightly lower intensities that are about 1.7 times lower than the $15 / 75 / 360 \mu \mathrm{m}$ PTEs at $200 \mathrm{~nL} / \mathrm{min}$ are observed with the $8 / 50 / 360 \mu \mathrm{m}$ PTEs. Finally, the lowest intensities for the PTEs ( 5.3 times lower at $200 \mathrm{~nL} / \mathrm{min})$ are observed in the plot for the 5/20/360 $\mu \mathrm{m}$ PTEs. This result is unexpected, because in many previous reports the smaller orifice i.d. emitters produced the greatest intensity signal [36-42]. In addition, the optimal intensities in this work are obtained at the higher flow rates, possibly indicating that even higher flow rates may produce greater signal intensity. However, as detailed in following sections, the ion utilization efficiency continually decreases as the flow rate is increased across the range tested. Additionally, the objective here is to study flow rates that are typically used in capillary HPLC and CE-MS.

The signal intensity produced by a commercially available monolithic emitter fabricated from potassium silicate polymerized inside a fused silica capillary was investigated. As shown in the first panel of Figure 5, the PST polytip sprays over a much smaller range of flow rates and ESI potentials compared with the other emitters. The signal is
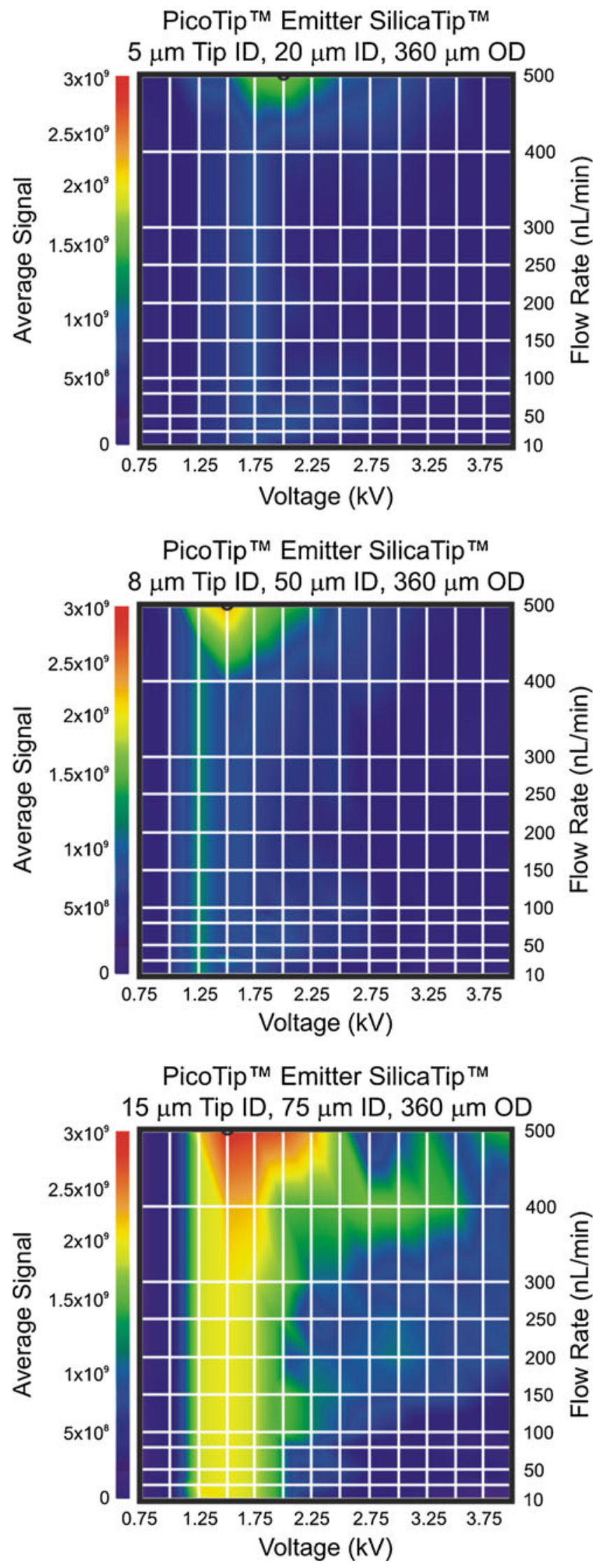

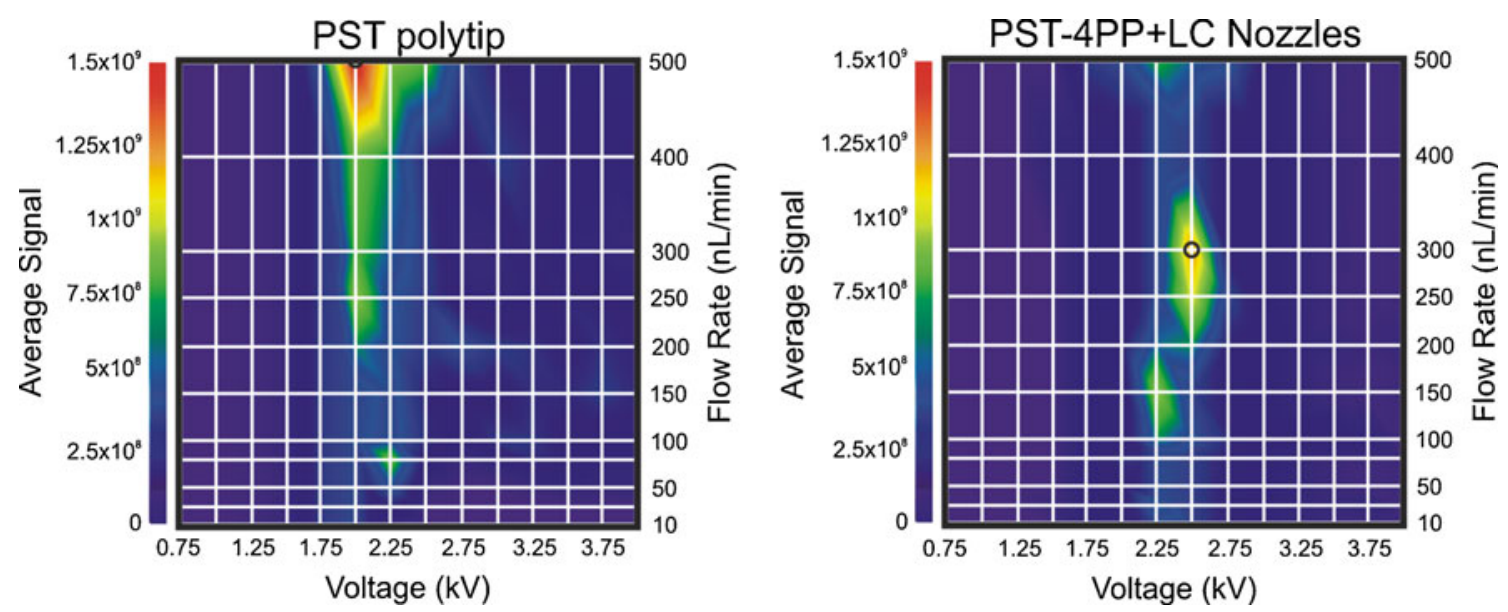

Figure 5. The 3D average intensity plots generated with the monolithic emitter and the molded polymeric nozzles are shown. The intensities for each plot are normalized to the highest intensities observed in the PST polytip 3D plot, which are significantly lower than the intensities observed with the EOEs. Again, the circled data points indicate the highest overall intensity for each emitter

substantially lower (about 6.8 times lower than the $50 \mu \mathrm{m}$ EOE tip at $200 \mathrm{~nL} / \mathrm{min}$ ) than the other tips over the lower flow rate ranges. Also, the maximum intensity was observed at a potential of $2.0 \mathrm{kV}$, which is slightly higher than the ESI voltage that produced maximum signal for other emitters. With these tips, the Taylor cone was frequently not anchored around the tip orifice and often expanded below the tip, which contributes to the reduced signal intensities observed compared with the other emitters.

A molded polypropylene tip (PST-4PP+LC Nozzle) that was attached to the end of a transfer capillary was also characterized. This polypropylene tip is similar in geometry to many emitters that are molded directly into microfluidic devices [58-65]. The polypropylene substrate imparts a very hydrophobic surface to this emitter compared with the other emitters characterized in this study. The results show that this emitter generated lower signal intensities than the other emitters and the signal intensity decreased significantly for

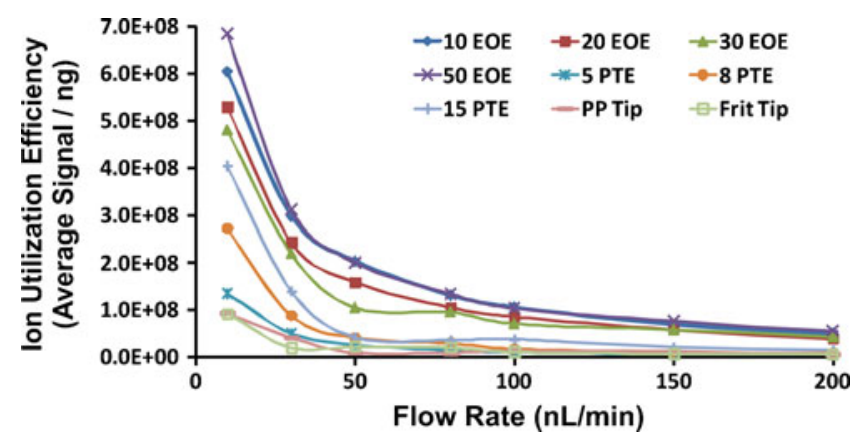

Figure 6. The ion utilization efficiency plots show the dependence of the signal intensity on the mass of sample consumed. As the flow rate is increased, the ratio of the signal intensity to mass of sample consumed decreases rapidly. The highest ion utilization efficiencies are achieved with the EOEs while the PTEs provide higher ion utilization efficiencies than the monolithic emitter and the polypropylene nozzle flow rates $\geq 400 \mathrm{~nL} / \mathrm{min}$ and for ESI potentials $\geq 2.75 \mathrm{kV}$. The highest intensity signals observed with this emitter were only obtained over a very limited flow rate range, 250 to $300 \mathrm{~nL} / \mathrm{min}$, and at a spray voltage of $2.5 \mathrm{kV}$. For the emitters, shown in Figure 5, the average signal intensities are comparable to those observed with the 5/20/360 $\mu \mathrm{m}$ PTE, however the average signal intensities are still lower than those observed with the EOEs.

For many of the investigated emitters the signal intensity increases with increasing flow rate, although the increase in signal intensity is relatively small compared with the increase in sample used. At higher flow rates ESI-MS behaves as a concentration sensitive system, but at lower flow rates the signal intensity becomes mass dependent [7, 21, 49]. In our studies, the increase in signal with increasing flow rate indicates the system is below the regime where it is entirely concentration sensitive, particularly at the lower end of the flow rate range used. At the lowest flow rates, the intensity does increase as the flow rates increases, but the increase in the signal intensity is less than directly proportional to the sample consumed. At the optimum ESI voltage, initially the intensity quickly increases as the flow rate is increased and then the rate of increase in signal intensity decreases near $200 \mathrm{~nL} / \mathrm{min}$. In general, the signal of the EOEs decreases at the highest flow rates. Such decreases in signal intensity have been observed previously at high flow rates $[7,21,32,43,46,49,51,53,66]$. The behavior of the PTEs is somewhat different as the signal intensity continually increased, although the slope continually decreases as the flow rate is increased over the flow rate range investigated. The two polymer emitters are characterized by peaks in the $3 \mathrm{D}$ plots, which indicate a marked decrease in signal as the flow rate is increased to the 300 and $400 \mathrm{~nL} / \mathrm{min}$ range.

For mass-limited samples, ion utilization plots [32] are more useful in method development than evaluation of the raw signal intensity. Therefore, 3D ion utilization plots were constructed from the data shown in Figures 3, 4, and 5 by dividing the signal intensity by the mass of sample 
consumed at the flow rate used for sample delivery. The 3D ion utilization plots have many similarities with the $3 \mathrm{D}$ plots of the signal intensity and therefore are not shown. The signal/mass, or the ion utilization efficiency, is shown as a function of flow rate in Figure 6. The displayed data was extracted from the 3D ion utilization efficiency plots at the optimum electrospray voltage for each emitter and clearly show that the most efficient generation of signal is observed at the lowest flow rate used $(10 \mathrm{~nL} / \mathrm{min})$ and the ion utilization decreases monotonically as the flow rate increases [32]. These results confirm that the most efficient signal generation for mass-limited samples is achieved at the lowest flow rates, which is $10 \mathrm{~nL} / \mathrm{min}$ for this study. As the flow rate is increased past $80 \mathrm{~nL} / \mathrm{min}$, little increase in signal is observed as the flow rate increases, and consequently the ion utilization efficiency decreases. Past $150 \mathrm{~nL} / \mathrm{min}$, the signal/mass becomes asymptotic, and the signal/mass is nearly completely dependent on the sample concentration and the dependence on the mass is negligible. The results presented here consistently show that the EOEs generate the greatest signal intensities and the best ion utilization. Furthermore, the EOEs produced higher values over a greater range of ESI voltages and flow rates than other emitters. Therefore, if the sample mass is limited, using an EOE, constraining the sample to a smaller volume, and decreasing the flow rate will improve the signal intensity.

For the analysis of mass-limited samples in which the signal must be maximized, the following plan provides a strategy for determining the best experimental settings. To begin, both the mass of the sample and the time needed for the MS analysis should be determined first. Of course, the time required for MS analysis can be variable depending on the applications and the experimenter can determine whether the longest analysis time or a shorter time should be considered. The next step is to determine what flow rate will provide the greatest signal. The ion utilization plots indicate that the lowest flow rate provides the most efficient signal, which was $10 \mathrm{~nL} / \mathrm{min}$ for the results reported here. Once the flow rate is set, a target volume of solution can be determined from the product of the analysis time and the flow rate. If the volume of solution is greater than the target volume, the sample should be enriched for the most efficient use of sample. If enrichment is not possible, small gains in signal intensity can be achieved by increasing the flow rate. However, this strategy assumes that the emitter is functioning near the concentration limit, as observed in the data presented herein.

Microfluidic devices are well suited for handling samples of small volume in solution and their architecture minimizes sample contamination and lends itself to automation. Consequently, numerous researchers have developed systems for coupling microfluidic devices with ESI-MS. Similar to their larger scale counter parts microfluidic separations and sample transport can be divided into 2 categories: (1) electrokinetic and (2) hydrodynamic or pressure driven mass transport. Hydrodynamic separations, such as HPLC, have been readily integrated into capillary and microchip platforms and their widespread use has been adopted $[7,56,67]$. EOF originates from the movement of the diffuse layer in an electric field and has the advantage of being able to provide plug-like flow that reduces band broadening greatly compared with pressure driven systems. However, constrictions in the capillary i.d., such as those presented by an emitter tip, can cause a parabolic flow profile to be superimposed on top of the plug-like flow profile, sacrificing much or all of the gains in bandwidth achieved with the electrokinetic system [23]. Even a discontinuous surface charge on the capillary or microchannel wall can lead to such broadening [68-72]. Although greater band broadening occurs in hydrodynamic systems than high performance electrokinetic systems, pressure driven systems are commonly used due to their robust nature. With pressure driven systems, the contribution to band broadening caused by the tapered electrospray tip is normally insignificant. In contrast, the performance of electrokinetic systems, such as $\mathrm{ME}$, can suffer greatly from the backpressure created by forcing the solution through a small orifice. In spite of the significant number of designs reported, the lack of an ideal interface with ESI has prevented CE and ME-MS from becoming widely adopted. Electrokinetic systems rely on EOF to create a bulk flow of solution. For ESI, the bulk flow must be non-zero and great enough to provide adequate solution for droplet formation. Therefore, the emitter plays a key role in the performance of CE and ME-MS systems with larger emitter orifices that are consistent with the capillary or microchannel i.d. inducing the least band broadening.

\section{Conclusions}

The performance of a number of commonly used electrospray emitters are evaluated for pressure driven flow. The signal intensity is compared for EOEs, PTEs, a monolithic, and a molded polypropylene emitter, over a range of flow rates and electrospray voltages. For the EOE and PTE emitters, the signal intensity exhibited only a very small dependence on the emitter i.d., and contrary to many previously reported results, the larger i.d. orifices studied provided slightly greater signal intensity. This result is important for electrokinetic systems because smaller i.d. emitters have deleterious effects on sample transport and sample delivery to the MS. However, it is important to note that many of the previous studies were preformed with nanospray systems that are not pressure driven. It is postulated that the difference in flow is largely responsible for the trend toward greater signal intensity for the larger emitters used. The intensity of the signal generated by the electrospray is found to be largely concentration-sensitive, but over the lower range of the flow rates investigated, some dependence on flow rate and the mass of sample consumed is observed. The ion utilization efficiency plots are consistent with previous reports and show a decrease in ion utilization efficiency as the flow rate is increased. The highest ion utilization efficiency is observed at the lowest flow rate used in this study, which is $10 \mathrm{~nL} / \mathrm{min}$. The EOEs provided the greatest signal intensities and it is speculated that the geometry of the EOE emitters play an important role in these gains as the EOEs are excellent at 
supporting a well-defined Taylor cone. These results can be broadly applied to numerous electrospray systems that utilize pressure driven flow.

\section{Acknowledgments}

The authors acknowledge that the research was performed at West Virginia University, and thank WVU and the WVNano program for support. The authors also acknowledge that this work is funded in part through NSF RII award EPS 0554328, for which the WV EPSCoR Office and the WVU Research Corp. provided matching funds. We would like to thank Akos Vertes for enlightening discussions about ESI.

\section{References}

1. Garza, S., Moini, M.: Analysis of complex protein mixtures with improved sequence coverage using $(\mathrm{CE}-\mathrm{MS} / \mathrm{MS})^{n}$. Anal. Chem. 78, 7309-7316 (2006)

2. Moini, M.: Capillary electrophoresis mass spectrometry and its application to the analysis of biological mixtures. Anal. Bioanal. Chem. 373, 466-480 (2002)

3. Xie, J., Miao, Y., Shih, J., Tai, Y.-C., Lee, T.D.: Microfluidic platform for liquid chromatography-tandem mass spectrometry analyses of complex peptide mixtures. Anal. Chem. 77, 6947-6953 (2005)

4. Licklider, L., Wang, X.Q., Desai, A., Tai, Y.C., Lee, T.D.: A micromachined chip-based electrospray source for mass spectrometry. Anal. Chem. 72, 367-375 (2000)

5. Meng, F., Cargile, B.J., Patrie, S.M., Johnson, J.R., McLoughlin, S.M., Kelleher, N.L.: Processing complex mixtures of intact proteins for direct analysis by mass spectrometry. Anal. Chem. 74, 2923-2929 (2002)

6. Amirkhani, A., Wetterhall, M., Nilsson, S., Danielsson, R., Bergquist, J.: Comparison between different sheathless electrospray emitter configurations regarding the performance of nanoscale liquid chromatography-time-of-flight mass spectrometry analysis. J. Chromatogr. A 1033, 257-266 (2004)

7. Abian, J., Oosterkamp, A.J., Gelpi, E.: Comparison of conventional, narrow-bore, and capillary liquid chromatography/mass spectrometry for electrospray ionization mass spectrometry: practical considerations. J. Mass Spectrom. 34, 244-254 (1999)

8. Voyksner, R.D., Lee, H.: Improvements in LC/electrospray ion trap mass spectrometry performance using an off-axis nebulizer. Anal. Chem. 71, 1441-1447 (1999)

9. Shen, Y., Zhao, R., Berger, S.J., Anderson, G.A., Rodriguez, N., Smith, R.D.: High-efficiency nanoscale liquid chromatography coupled on-line with mass spectrometry using nanoelectrospray ionization for proteomics. Anal. Chem. 74, 4235-4249 (2002)

10. Sanz-Nebot, V., Balaguer, E., Benavente, F., Barbosa, J.: Comparison of sheathless and sheath-flow electrospray interfaces for the capillary electrophoresis-electrospray ionization-mass spectrometry analysis of peptides. Electrophoresis 26, 1457-1465 (2005)

11. Wahl, J.H., Gale, D.C., Smith, R.D.: Sheathless capillary electrophoresis-electrospray ionization mass spectrometry using $10 \mathrm{~mm}$ i.d. Capillaries: Analysis of tryptic digests of cytochrome c. J. Chromatogr. A 659, 217-222 (1994)

12. Foret, F., Thompson, T.J., Vouros, P., Karger, B.L., Gebauer, P., Bocek, P.: Liquid sheath effects on the separation of proteins in capillary electrophoresis/electrospray mass spectrometry. Anal. Chem. 66, 4450-4458 (1994)

13. Huikko, K., Kotiaho, T., Kostiainen, R.: Effects of nebulizing and drying gas flow on capillary electrophoresis/mass spectrometry. Rapid Commun. Mass Spectrom. 16, 1562-1568 (2002)

14. Smith, R.D., Barinaga, C.J., Udseth, H.R.: Improved electrospray ionization interface for capillary zone electrophoresis-mass spectrometry. Anal. Chem. 60, 1948-1952 (1988)

15. Whitt, J.T., Mehdi, M.: Capillary electrophoresis to mass spectrometry interface using a porous junction. Anal. Chem. 75, 2188-2191 (2003)

16. Axén, J., Axelsson, B.-O., Jörntén-Karlsson, M., Petersson, P., Sjöberg, P.J.R.: An investigation of peak-broadening effects arising when combining CE with MS. Electrophoresis 28, 3207-3213 (2007)
17. Nilsson, S., Markides, K.E.: On-column conductive coating for thermolabile columns used in capillary zone electrophoresis sheathless electrospray ionization mass spectrometry. Rapid Commun. Mass Spectrom. 14, 6-11 (2000)

18. Kelly, J.F., Ramaley, L., Thibault, P.: Capillary zone electrophoresiselectrospray mass spectrometry at submicroliter flow rates: Practical considerations and analytical performance. Anal. Chem. 69, 51-60 (1997)

19. Hsieh, F., Baronas, E., Muir, C., Martin, S.A.: A novel nanospray capillary zone electrophoresis/mass spectrometry interface. Rapid Commun. Mass Spectrom. 13, 67-72 (1999)

20. Moseley, M.A., Jorgenson, J.W., Shabanowitz, J., Hunt, D.F., Tomer, K. B.: Optimization of capillary zone electrophoresis/electrospray ionization parameters for the mass spectrometry and tandem mass spectrometry analysis of peptides. J. Am. Soc. Mass Spectrom. 3, 289-300 (1992)

21. Wahl, J.H., Goodlett, D.R., Udseth, H.R., Smith, R.D.: Use of smalldiameter capillaries for increasing peptide and protein detection sensitivity in capillary electrophoresis-mass spectrometry. Electrophoresis 14, 448-457 (1993)

22. Mellors, J.S., Gorbounov, V., Ramsey, R.S., Ramsey, J.M.: Fully integrated glass microfluidic device for performing high-efficiency capillary electrophoresis and electrospray ionization mass spectrometry. Anal. Chem. 80, 6881-6887 (2008)

23. Lazar, I.M., Ramsey, R.S., Jacobson, S.C., Foote, R.S., Ramsey, J.M.: Novel microfabricated device for electrokinetically induced pressure flow and electrospray ionization mass spectrometry. J. Chromatogr. A 892, 195-201 (2000)

24. Zhang, B., Foret, F., Karger, B.L.: A microdevice with integrated liquid junction for facile peptide and protein analysis by capillary electrophoresis/ electrospray mass spectrometry. Anal. Chem. 72, 1015-1022 (2000)

25. Zhang, B., Liu, H., Karger, B.L., Foret, F.: Microfabricated devices for capillary electrophoresis-electrospray mass spectrometry. Anal. Chem. 71, 3258-3264 (1999)

26. Tachibana, Y., Otsuka, K., Terabe, S., Arai, A., Suzuki, K., Nakamura, S.: Robust and simple interface for microchip electrophoresis-mass spectrometry. J. Chromatogr. A 1011, 181-192 (2003)

27. Ramsey, R.S., Ramsey, J.M.: Generating electrospray from microchip devices using electroosmotic pumping. Anal. Chem. 69, 1174-1178 (1997)

28. Lazar, I.M., Li, L., Yang, Y., Karger, B.L.: Microfluidic device for capillary electrochromatography-mass spectrometry. Electrophoresis 24, 3655-3662 (2003)

29. Akashi, S., Suzuki, K., Arai, A., Yamada, N., Suzuki, E.-I., Hirayama, K., Nakamura, S., Nishimura, Y.: Top-down analysis of basic proteins by microchip capillary electrophoresis mass spectrometry. Rapid Commun. Mass Spectrom. 20, 1932-1938 (2006)

30. Wahl, J.H., Smith, R.D.: Comparison of buffer systems and interface designs for capillary electrophoresis-mass spectrometry. J. Capillary Electrophoresis 001, 62-71 (1994)

31. Marginean, I., Kelly, R.T., Page, J.S., Tang, K., Smith, R.D.: Electrospray characteristic curves: In pursuit of improved performance in the nanoflow regime. Anal. Chem. 79, 8030-8036 (2007)

32. Marginean, I., Kelly, R.T., Prior, D.C., LaMarche, B.L., Tang, K., Smith, R.D.: Analytical characterization of the electrospray ion source in the nanoflow regime. Anal. Chem. 80, 6573-6579 (2008)

33. Kebarle, P., Ho, Y.: On the mechanism electrospray mass spectrometery. In: Cole, R.B. (ed.) Electrospray ionization mass spectrometry fundamentals, instrumentation, and applications, pp. 3-63. John Wiley and Sons, Inc, New York (1997)

34. Tojo, H.: Properties of an electrospray emitter coated with material of low surface energy. J. Chromatogr. A 1056, 223-228 (2004)

35. Shui, W., Yu, Y., Xu, X., Huang, Z., Xu, G., Yang, P.: Microelectrospray with stainless steel emitters. Rapid Commun. Mass Spectrom. 17, 1541-1547 (2003)

36. Wilm, M.S., Mann, M.: Electrospray and taylor-cone theory, dole's beam of macromolecules at last? Int. J. Mass Spectrom. Ion Processes 136, 167-180 (1994)

37. Wilm, M.S., Mann, M.: Analytical properties of the nanoelectrospray ion source. Anal. Chem. 68, 1-8 (1996)

38. Fernandez de la Mora, J., Loscertales, I.G.: The current emitted by highly conducting taylor cones. J. Fluid Mech 260, 155-184 (1994)

39. de Juan, L., Fernandez de la Mora, J.: Charge and size distributions of electrospray drops. J. Colloid Interface Sci. 186, 280-293 (1997)

40. Ek, P., Sjodahl, J., Roeraade, J.: Electrospray ionization from a gap with adjustable width. Rapid Commun. Mass Spectrom. 20, 3176-3182 (2006) 
41. Li, Y., Cole, R.B.: Shifts in peptide and protein charge state distributions with varying spray tip orifice diameter in nanoelectrospray fourier transform ion cycltron resonance mass spectrometry. Anal. Chem. 75, 5739-5746 (2003)

42. Nemes, P., Marginean, I., Vertes, A.: Spraying mode effect on droplet formation and ion chemistry in electrosprays. Anal. Chem. 79, 3105$3116(2007)$

43. Ishihama, Y., Katayama, H., Asakawa, N., Oda, Y.: Highly robust stainless steel tips as microelectrospray emitters. Rapid Commun. Mass Spectrom. 16, 913-918 (2002)

44. Kelly, R.T., Page, J.S., Luo, Q., Moore, R.J., Orton, D.J., Tang, K., Smith, R.D.: Chemically etched open tubular and monolithic emitters for nanoelectrospray ionization mass spectrometry. Anal. Chem. 78, 7796-7801 (2006)

45. Tang, L., Kebarle, P.: Effect of the conductivity of the electrosprayed solution on the electrospray current. Factors determining analyte sensitivity in electrospray mass spectrometry. Anal. Chem. (Washington, DC) 63, 2709-2715 (1991)

46. Page, J.S., Kelly, R.T., Tang, K., Smith, R.D.: Ionization and transmission efficiency in an electrospray ionization-mass spectrometry interface. J. Am. Soc. Mass Spectrom. 18, 1582-1590 (2007)

47. Tang, X., Bruce, J.E., Hill, J.H.H.: Characterizing electrospray ionization using atmospheric pressure ion mobility spectrometry. Anal. Chem. 78, 7751-7760 (2006)

48. Emmett, M.R., Caprioli, R.M.: Micro-electrospray mass spectrometry: Ultra-high-sensitivity analysis of peptides and proteins. J. Am. Soc. Mass Spectrom. 5, 605-613 (1994)

49. Tang, K., Page, J.S., Smith, R.D.: Charge competition and the linear dynamic range of detection in electrospray ionization mass spectrometry. J. Am. Soc. Mass Spectrom. 15, 1416-1423 (2004)

50. Schmidt, A., Karas, M., Dulcks, T.: Effect of different solution flow rates on analyte ion signals in Nnano-ESI MS, or: When Does ESI Turn into Nano-ESI? J. Am. Soc. Mass Spectrom. 14, 492-500 (2003)

51. Ikonomou, M.G., Blades, A.T., Kebarle, P.: Electrospray-ion spray: A comparison of mechanisms and performance. Anal. Chem. (Washington, DC), 63, 1989-1998 (1991).

52. Oosterkamp, A.J., Gelpi, E., Abian, J.: quantitative peptide bioanalysis using column-switching nano liquid chromatography/mass spectrometry. J. Mass Spectrom. 33, 976-983 (1998)

53. Hopfgartner, G., Wachs, T., Bean, K., Henion, J.: High-flow ion spray liquid chromatography/mass spectrometry. Anal. Chem. (Washington, DC) 65, 439-446 (1993)

54. Valaskovic, G.A., Kelleher, N.L., Little, D.P., Aaserud, D.J., McLafferty, F.W.: Attomole-sensitivity electrospray source for large-molecule mass spectrometry. Anal. Chem. 67, 3802-3805 (1995)

55. Giddings, J.C.: Flow in Capillaries. In: Unified Separation Science, pp. 58-61. John Wiley and Sons, Inc. (1991).

56. Cortes, H.J., Pfeiffer, C.D., Richter, B.E., Stevens, T.S.: Porous ceramic bed supports for fused silica packed capillary columns used in liquid chromatography. J. High Resolut. Chromatogr.- Chromatography Commun 10, 446-448 (1987)
57. Valaskovic, G.A., Murphy, J.P., Lee, M.S.: Automated orthogonal control system for electrospray ionization. J. Am. Soc. Mass Spectrom. 15, 1201-1215 (2004)

58. Kim, J.S., Knapp, D.R.: Microfabricated PDMS multichannel emitter for electrospray ionization mass spectrometry. J. Am. Soc. Mass Spectrom. 12, 463-469 (2001)

59. Kim, J.S., Knapp, D.R.: Microfabrication of polydimethylsiloxane electrospray ionization emitters. J. Chromatogr. A 924, 137-145 (2001)

60. Kim, J.-S., Knapp, D.R.: Miniaturized multichannel electrospray ionization emitters on poly(dimethylsiloxane) microfluidic devices. Electrophoresis 22, 3993-3999 (2001)

61. Bedair, M.F., Oleschuk, R.D.: Fabrication of porous polymer monoliths in polymeric microfluidic chips as an electrospray emitter for direct coupling to mass spectrometry. Anal. Chem. 78, 1130-1138 (2006)

62. Thorslund, S., Lindberg, P., Andren, P.E., Nikolajeff, F., Bergquist, J.: Electrokinetic-driven microfluidic system in poly(Dimethylsiloxane) for mass spectrometry detection integrating sample injection, capillary electrophoresis, and electrospray emitter on-chip. Electrophoresis 26, 4674-4683 (2005)

63. Kelly, R.T., Tang, K., Irimia, D., Toner, M., Smith, R.D.: Elastomeric microchip electrospray emitter for stable cone-jet mode operation in the nanoflow regime. Anal. Chem. (Washington, DC) 80, 3824-3831 (2008)

64. Kim, J.-S., Knapp, D.R.: Microfabricated multichannel electrospray ionization emitters on polydimethylsiloxane (PDMS) microfluidic devices. Miniaturization Mass Spectrom, 67-96 (2009).

65. Iannacone, J.M., Jakubowski, J.A., Bohn, P.W., Sweedler, J.V.: A multilayer poly(Dimethylsiloxane) electrospray ionization emitter for sample injection and online mass spectrometric detection. Electrophoresis 26, 4684-4690 (2005)

66. Hopfgartner, G., Bean, K., Henion, J., Henry, R.: Ion spray mass spectrometric detection for liquid chromatography: A concentration- or a mass-flow-sensitive device? J. Chromatogr. 647, 51-61 (1993)

67. Smyth, W.F., Brooks, P.: A critical evaluation of high performance liquid chromatography-electrospray ionization-mass spectrometry and capillary electrophoresiselectrospray-mass spectrometry for the detection and determination of small molecules of significance in clinical and forensic science. Electrophoresis 25, 1413-1446 (2004)

68. Shariff, K., Ghosal, S.: Peak tailing in electrophoresis due to alteration of the wall charge by adsorbed analytes a numerical simulations and asymptotic theory. Anal. Chim. Acta 507, 87-93 (2004)

69. Ghosal, S., Lu, Z.: Electroosmotic flow and zone broadening in microuidic channels of variable cross-section and wall charge. Proceedings of the 2002 International Conference on Modeling and Simulation of Microsystems; San Juan, Puerto Rico, (2002).

70. Ghosal, S.: The effect of wall interactions in capillary-zone electrophoresis. J. Fluid Mech. 491, 285-300 (2003)

71. Ghosal, S.: Lubrication theory for electroosmotic flow in a microfluidic channel of slowly varying cross-section and wall charge. J. Fluid Mech. 459, 103-128 (2002)

72. Ghosal, S.: Band broadening in a microcapillary with a stepwise change in the $\zeta$-potential. Anal. Chem. 74, 4198-4203 (2002) 